\title{
artigo
}

\section{Homens que fazem sexo com homens: conhecimento, empoderamento a profilaxia pré- exposição ao vírus da imunodeficiência humana}

Men who have sex with men: knowledge, empowerment for human immunodeficiency virus pre-exposure prophylaxis Hombres que tienen sexo con hombres: conocimiento, empoderamiento para la profilaxis previa a la exposición al virus de la inmunodeficiencia humana

\begin{abstract}
RESUMO
Objetivo: Analisar o conhecimento e empoderamento de homens que fazem sexo com homens sobre a profilaxia pré-exposição ao Vírus da imunodeficiência Humana. Método: Trata-se de revisão integrativa. Foi realizado um levantamento no banco de dados da Biblioteca Virtual em Saúde, em suas respectivas bases Literatura Latino-Americana e do Caribe em Ciências da Saúde e Medical Literature Analysis and Retrieval System Online. Utilizou os descritores cadastrados no Portal de Descritores das Ciências da Saúde (DeCS) e (MESH): "pre-exposure prophylaxis for hiv" AND "men who have sex with men" OR "gay" OR "homossexual" OR "msm" com os operadores booleanos AND e OR. Resultados: Dos 325 estudos encontrados, três responderem a pergunta da pesquisa. Conclusão: A disponibilidade de alguns métodos preventivos beneficiam, em âmbito individual, a proteção nos diferentes momentos da cadeia de transmissão do HIV e seu uso complementar promete avanços consistentes na resposta à epidemia com impacto na redução da incidência.
\end{abstract}

DESCRITORES: Prevenção; Homens que fazem sexo com Homens; Conhecimento; Homossexual.

\begin{abstract}
Objective: To analyze the knowledge and empowerment of men who have sex with men about Human Immunodeficiency Virus pre-exposure prophylaxis. Method: This is an integrative review. A survey was conducted in the database of the Virtual Health Library, in their respective databases Latin American and Caribbean Health Sciences and Medical Literature Analysis and Retrieval System Online. It used the descriptors registered in the Health Sciences Descriptors Portal (DeCS) and (MESH): "pre-exposure prophylaxis for hiv" AND "men who have sex with men" OR "gay" OR "homosexual" OR "msm" with boolean operators AND and OR. Results: Of the 325 studies found, 03 answered the research question. Conclusion: The availability of some preventive methods individually benefits the protection at different times of the HIV transmission chain and their complementary use promises consistent advances in response to the epidemic with an impact on reducing incidence.
\end{abstract}

DESCRIPTORS: Pre Exposure Prophylaxis; Men who have sex with men; Knowledge; Homosexual.

\section{RESUMEN}

Objetivo: analizar el conocimiento y el empoderamiento de los hombres que tienen sexo con hombres sobre la profilaxis previa a la exposición del virus de la inmunodeficiencia humana. Método: esta es una revisión integradora. Se realizó una encuesta en la base de datos de la Biblioteca Virtual en Salud, en sus respectivas bases de datos en línea del Sistema de Análisis y Recuperación de Literatura Médica y Ciencias de la Salud de América Latina y el Caribe. Se utilizaron los descriptores registrados en el Portal de Descriptores de Ciencias de la Salud (DeCS) y (MESH): "profilaxis previa a la exposición para el VIH" Y "hombres que tienen sexo con hombres" O "gay" O "homosexual" O "msm" con operadores booleanos AND y OR. Resultados: De los 325 estudios encontrados, 03 respondieron la pregunta de investigación. Conclusión: La disponibilidad de algunos métodos preventivos beneficia individualmente la protección en diferentes momentos de la cadena de transmisión del VIH y su uso complementario promete avances consistentes en respuesta a la epidemia con un impacto en la reducción de la incidencia.

DESCRIPTORES: profilaxis preexposición; Hombres que tienen sexo con hombres; Conocimiento; Homosexual.

RECEBIDO EM: 27/01/2021 APROVADO EM: 09/02/2021 
Esther Amorim Ouriques de Ataíde

Enfermeira. Mestranda em enfermagem - UPE.

ORCID: 0000-0003-2678-4563

Carla Andreia Alves de Andrade

Professora Substituta da UFPE-CAV. Enfermeira. Doutoranda em enfermagem -UPE.

ORCID: 0000-0002-8877-3344

Daniela de Aquino Freire

Enfermeira. Doutoranda em enfermagem - UPE.

ORCID: 0000-0002-6708-5139

\section{Fátima Maria da Silva Abrão}

Livre Docente pela Universidade de Pernambuco. Doutora em Enfermagem pela Universidade de São Paulo/Escola de Enfermagem de Ribeirão Preto (USP/EERP).

ORCID: 0000-0003-3254-2851

\section{INTRODUÇÃO}

$\mathbf{N}$ o inicio dos anos 80, o vírus da Imunodeficiência humana - HIV foi evidenciado como uma elevada epidemia preocupando e mobilizando as autoridades de saúde naquela época ${ }^{1}$. Apontada e rotulada pelo preconceito, a Síndrome da Imunodeficiência Adquirida - Aids era vista como a doença dos 5H, ou seja, Homossexuais, Hemofílicos, Haitianos, Heroinômanos (usuários de heroína injetável) e Hookers (do inglês, profissionais do sexo) ${ }^{2}$.

Já nos anos 90, dados a nível internacional, indicavam que, após atingir o pico de mais de 3 milhões, a ocorrência mundial do HIV teria permanecido relativamente constante desde 2005, com mais de 2,5 milhões de novas infecções, anualmente. Em contrapartida, o número de pessoas que vivem com HIV mostra-se com uma tendência de crescimento, atingindo cerca de 38,8 milhões de pessoas em 2015, remetendo, assim, que a mortalidade decresça, acumulando, no mesmo ano, menos de 1,3 milhões de mortes ${ }^{3}$.

Dados epidemiológicos verifica ainda a ampliação dos casos quebrando assim a visão distorcida de que a AIDS restringia-se apenas a determinados "grupos de risco". Deste modo, passou-se a considerar que, independente da raça, idade, gênero, opção sexual e condição socioeconômica, qualquer pessoa está propensa à exposição ao vírus, passando a ser empregado o conceito de vulnerabilidade, no qual compreende si- tuações que alguém se expõe numa situação de adoecimento e/ou debilidade 2 .

Atualmente, no Brasil, os Homens que fazem Sexo com Homens (HSH) são uma das categorias mais afetadas pelo HIV. Estratégias têm sido aplicadas para o enfrentamento da epidemia, como o envolvimento da sociedade civil e do governo em ações comunitárias de prevenção para os HSH e de desestigmatização das homossexualidades na sociedade ${ }^{4}$. Trazendo assim, medidas de promoção a saúde com distribuição gratuita de preservativos; acessibilidade a profilaxia pré-exposição (PREP), a testagem para a população em geral, e da terapia antirretroviral para as pessoas vivendo com HIV e Aids (PVHA) 5 .

Não obstante, enquanto a prevalência do HIV estimada para a população em geral (13 a 49 anos) é de $0,6 \%$ ( $0,8 \%$ para homens $)^{6}$. Um estudo realizado em 2008 e 2009 evidenciou que a prevalência para HSH em 10 cidades oscilou entre 5,2\% (Recife) e 23,4\% (Brasília), com média de $14,2 \%$ - duas e três vezes maior que a estimada para, respectivamente, mulheres trabalhadoras do sexo e usuários de drogas 7 . Dos 3.449 participantes do estudo, 36,5\% reportaram intercurso anal receptivo desprotegido (IARD) ${ }^{8}$.

Homens praticantes de IARD são sujeitos a maior risco de infecção que os que realizam intercurso anal insertivo desprotegido (IAID) ${ }^{9}$. Além disso, fatores como conhecimento escasso sobre a profilaxia pré-exposição ao HIV que tem sido um método de prevenção ao HIV evidenciam a vulnerabilidade dessa parcela populacional ${ }^{10}$.

Ainda que existam dados divulgados nos últimos 4 anos pelo Ministério da Saúde sobre o conhecimento de práticas preventivas ao HIV - sendo este o mais o comum o preservativo, mesmo assim, mais de $45 \%$ da população sexualmente ativa do país não o usa nas relações sexuais ${ }^{11}$.

Diante desse cenário, novas estratégias de prevenção surgem como ferramentas complementares no enfrentamento da epidemia de HIV ampliando a gama de opções que os indivíduos terão para se prevenir contra o vírus e oferecendo mais alternativas - cientificamente eficazes - em relação à única opção disponível até pouco tempo atrás: o preservativo $^{11}$. Entre as novas estratégias estacam-se o uso do tratamento como prevenção a Profilaxia Pré-exposição (PrEP).

A PrEP é um medicamento antirretroviral utilizado por aqueles indivíduos que não estão infectados pelo HIV, mas se encontram em situação de vulnerabilidade a infecção. Com a medicação circulante no sangue no momento de contato com vírus o HIV ficará impedido de se estabelecer no organismo ${ }^{12}$. A medicação consiste na combinação de dois medicamentos em um único comprimido: o fumarato de tenofovir desproxila (TDF - 300mg) e a emtricitabina (FTC - 200mg), e sua utilização é feita diariamente, por via oral ${ }^{12}$. No Brasil, a PrEP começou a ser disponibilizada pelo SUS em dezembro de 2017 e está disponível em 36 serviços de saúde em 22 cidades brasileiras. Sendo assim, os desafios inerentes 


\section{artigo}

Ataíde, E.A.O.; Andrade, C.A.A.; Freire, D.A.; Abrão, F.M.S.

Homens que fazem sexo com homens: conhecimento, empoderamento a profilaxia pré-exposição ao vírus da imunodeficiência humana

ao conhecimento e o empoderamento da utilização dessa profilaxia em homens que fazem sexo com homens potencializam o risco da infecção ao HIV, entretanto a maioria dos problemas evidenciados por essa população talvez esteja vinculada a necessidade de implementação de estratégias direcionadas em sua atual situação de saúde. Além disso, estereótipos socialmente construídos em relação à doença como também preconceitos em relação à orientação sexual ${ }^{12}$.Nesse sentido, ressalta-se a importância de se refletir e discutir a temática: Qual o conhecimento e empoderamento de Homens que fazem sexo com homens sobre à profilaxia pré-exposição ao HIV em seu contexto atual. Dessa forma, o estudo tem como objetivo analisar a partir das produções científicas o conhecimento e empoderamento de homens que fazem sexo com homens sobre a profilaxia pré-exposição ao HIV.

\section{MÉTODO}

Esta revisão integrativa seguiu todas as etapas preconizadas. Primeiramente, definiu-se o tema do estudo e o objetivo, emergindo a questão da pesquisa. Em seguida foi realizado a análise a partir das produçóes científicas sobre o conhecimento e empoderamento de homens que fazem sexo com homens sobre a profilaxia pré-exposição ao HIV. Para a elaboração da revisão, foram seguidas as seguintes etapas: Primeira Etapa: realizada busca online, mediante levantamento no banco de dados da Biblioteca Virtual em Saúde (BVS), em suas respectivas bases Literatura Latino-Americana e do Caribe em Ciências da Saúde (LILACS) e Medical Literature Analysis and Retrieval System Online (MEDLINE) em virtude do quantitativo da produção científica relativa à temática; na etapa seguinte houve a seleção dos descritores cadastrados no Portal de Descritores das Ciências da Saúde (DeCS) e (MESH): "pre-exposure prophylaxis for hiv" AND "men who have sex with men" OR "gay" OR "homossexual" OR "msm". Estes descritores foram combinados com os operadores booleanos AND e OR a fim de refinar os estudos de acordo com tema em questão. Em seguida, foram aplicados os seguintes filtros; textos completos disponíveis, base de dados nacionais e internacionais, assunto principal onde foi selecionado, profilaxia pré-exposição, hiv, homossexual masculino e homens que fazem sexo com homens, além dos idiomas em inglês, espanhol e português. A busca ocorreu em junho de 2019 a julho de 2019; dando sequência a terceira etapa, seguindo a recomendação PRISMA, seguindo o fluxograma da (Figura 1).

Dando prosseguimento, a etapa seguinte ocorreu por meio da extração de dados seguindo a orientação de um formulário estruturado e validado por Ursi $(2005)^{13}$, adaptado para as questôes norteadoras do presente estudo.

Com o objetivo de avaliar a qualidade metodológica dos artigos selecionados, aplicou-se o instrumento Critical Appraisal Skills Programme - CASP esse instrumento possui 10 itens que são pontuados, incluindo: 1 ) objetivo; 2) adequação do método; 3) apresentação dos procedimentos teóricos metodológicos; 4) critérios de seleção da amostra, 5) detalhamento da amostra; 6) relação entre pesquisadores e pesquisados; 7) respeito aos aspectos éticos; 8) rigor na análise dos dados; 9) propriedade para discutir os resultados e
10) contribuições e limitações da pesquisa. Onde ao final é considerado:

A (06 a 10 pontos) - A2, A3, A4. Estudos com boa qualidade metodológica e viés reduzido. B (no mínimo 05 pontos) - A1, A5. Estudos com qualidade metodológica satisfatória, mas com potencial de viés aumentado.

O segundo instrumento utilizado para avaliação do nível de evidência foi a Classificação Hierárquica das Evidências para Avaliação dos Estudos Stillwell 4 sendo classificados os estudos nos seguintes níveis: (I) revisão sistemática ou metanálise; (II) ensaios clínicos randomizados; (III) ensaio clínico sem randomização; (IV) estudos de coorte e de caso-controle; (V) revisão sistemática de estudos descritivos e qualitativos e (VI) único estudo descritivo ou qualitativo. Finalmente, a última etapa foi apresentada pelos estudos selecionados e categorizados por temáticas a fim de serem utilizados como base para subsidiar as reflexões acerca do objeto de estudo.

\section{RESULTADOS}

Do total de 325 estudos encontrados nas bases de dados, foram sintetizados em

\section{Figura 1- Fluxograma de Processo de seleção de artigos conforme escala PRISMA}

$1^{2}$ Etapa: Identificação dos artigos - Cruzamento dos descritores nas bases de dados da biblioteca virtual Saúde

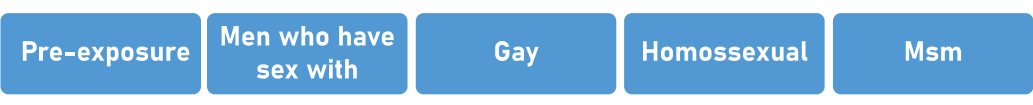

Total de 325 artigos selecionados

Aplicação dos filtros na bade de dados

$2^{\mathrm{a}}$ Etapa: Seleção dos artigos - Foram 05 Artigos selecionados na seguintes bases de dados

05 Medline 0 Lilacs

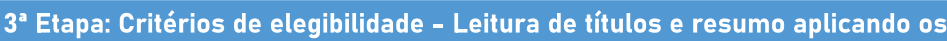
critérios de inclusão

03 Artigos selecionados na bases de dados

03 Medline 0 Lilacs

Leitura dos artigos na íntegra aplicando os críterios de exclusão

03 Medline 0 Lilacs

$4^{\mathrm{a}}$ Etapa: Inclusão de artigos

Amostra final: $\mathbf{0 3}$ artigos fizeram parte da revisão 
apenas três artigos por estes responderem a pergunta da pesquisa, vale ressaltar que os artigos foram no idioma inglês. Um ponto importante é que os artigos enquadraram-se, apenas, no recorte temporal de 2014 a 2018 e quanto à natureza desses, três estudos foram classificados como de pesquisa. Mostra-se que os estudos foram publicados no Alemanha, Estados Unidos e no Brasil.

Neles foram, também, classificados quanto ao nível de evidência, como mostra (Quadro 1).

Após a análise do conteúdo das publicaçôes, surgiu uma categoria com a seguinte temática: Profilaxia pré-exposição para homens e mulheres transexuais que fazem sexo com homens no Brasil: oportunidades e desafios.

\section{DISCUSSÃO}

Dos estudos desenvolvidos sobre o conhecimento de homens que fazem sexo com homens sobre o método de prevenção PrEP - profilaxia pré-exposição ao HIV, evidenciaram que houve redução na incidência de HIV, embora ainda existam muitos desafios a serem enfrentados acerca da temática, isso corrobora com o estudo de Ricardo Niklas Werner (2018) onde mostrou que em Berlim as infecções pelo HIV sofreram declínio pelo uso da PrEP e estava associada à acessibilidade através de canais de comunicação ${ }^{15}$. Nessa mesma pesquisa, os participantes se sentiram sabedores e bem informados, a média de idade dos participantes foi de 37,4 anos, intervalo 18-79. A maioria relatou ter relações sexuais sem preservativos com dois ou mais parceiros nos últimos seis meses e que se sentiam preocupados com a falta informações sobre o uso da $\mathrm{PrEP}^{15}$.

No estudo de Alia A. Al-Tayyib (2014) além do conhecimento entre homens que fazem sexo com homens avaliou-se também a disposição para usar a PrEP, nessa pesquisa comparou-se um estudo examinando o conhecimento da PrEP em 2008 e em 2011, os resultados não foram satisfatórios, pois menos de $30 \%$ dos participantes relataram não conhecer a profilaxia pré-exposição ${ }^{16}$.

Nos dois estudos citados, anteriormente, é notório que fatores associados ao conhecimento escasso sobre a PrEP ao HIV reforçam ainda mais a vulnerabilidade de desse populacional ${ }^{11}$. Tal resultado pode estar associado à ineficiência da promoção em saúde que faz parte do processo de educação ofertada pelos profissionais de saúde. Ressalta-se, portanto, a necessidade contínua de suporte necessário dos profissionais envolvidos nesse processo e sua compreensão acerca da necessidade de conhecer e disseminar este cuidado específico.

Um estudo que mereceu destaque foi o de Brenda Hoaglan (2017) que além do conhecimento de homens que fazem sexo com homens sobre a Profilaxia Pré-exposição ao HIV, procurou-se também analisar as oportunidades e desafios que a terapia vem encarando no Brasil. Nele, os principais aspectos da epidemia da Aids entre homens que fazem sexo com homens e a abrangente resposta a epidemia são apresentados por todo território nacional ${ }^{17}$.

Nesse artigo, ainda, o acesso universal aos serviços de saúde prestados pelo Sistema Único de Saúde (SUS) e a gama de serviços de prevenção e cuidados são disponíveis em todo país para indivíduos com HIV positivos e aqueles que possuem risco a infecção,

\section{Quadro 1 - Distribuição dos artigos analisados por título/ano, autor, objetivo, tipo de estudo e principais conclusões} do estudo. Recife - PE, 2019.

\begin{tabular}{|c|c|c|c|c|}
\hline ARTIGOS & TÍTULO/ANO/AUTOR & OBJETIVO DO ESTUDO & $\begin{array}{c}\text { DESENHO } \\
\text { METODOLÓGICO/NIVEL } \\
\text { E EVIDÊNCIA DO ESTUDO }\end{array}$ & CONCLUSÃO/DESFECHO \\
\hline 01 & $\begin{array}{l}\text { Knowledge and use of HIV } \\
\text { pre-exposure prophylaxis } \\
\text { among men in Berlin - } \\
\text { multicenre. cross secional } \\
\text { survey/2018/Ricardo Niklas } \\
\text { Werner et. al. }\end{array}$ & $\begin{array}{l}\text { Insvestigar o conhecimento } \\
\text { e o uso da profilaxa pre-ex- } \\
\text { posição entre homens que } \\
\text { fazem sexo com homens em } \\
\text { Berlin e fatores preditivos do } \\
\text { desejo de usar a profilaxia e o } \\
\text { histórico da meicação }\end{array}$ & $\begin{array}{c}\text { Estudo quantitativo trans- } \\
\text { versal/CASP: A1 Nível de } \\
\text { evidência: VI }\end{array}$ & $\begin{array}{c}\text { Foi encontrado alta conscen- } \\
\text { tização da PrEP entre homens } \\
\text { que fazem sexo com homens, } \\
\text { mas também uma forte } \\
\text { necessidade de educação } \\
\text { continuada sobre a temática }\end{array}$ \\
\hline 02 & $\begin{array}{l}\text { Knowledge of pre-exposure } \\
\text { Prophylaxis (PrEP) for HIV } \\
\text { prevention among men who } \\
\text { have sex with men in Denver, } \\
\text { Colorado/2014/ Alia A. Al- } \\
\text {-tayyio et. al. }\end{array}$ & $\begin{array}{l}\text { Avaliar o conhecimneto da } \\
\text { profilaxia pre-exposição - } \\
\text { PrEP e disposição para usar } \\
\text { a terapia }\end{array}$ & $\begin{array}{c}\text { Estufo quantitativo, } \\
\text { descritivo/CASP: A1 Nível } \\
\text { de evidência: V1 }\end{array}$ & $\begin{array}{l}\text { Poucos homens que fazem } \\
\text { sexo com homens tem } \\
\text { conhecimento da profilaxia } \\
\text { pre-exposição ao HIV. sendo } \\
\text { necessária a inclusão da } \\
\text { educação como forma e } \\
\text { prevenção }\end{array}$ \\
\hline 03 & $\begin{array}{c}\text { Pre-exposure prophylaxis } \\
\text { for men and transgender } \\
\text { women who have sex with } \\
\text { men in Brazil: Oppotunities } \\
\text { and challenges/2017/ Brenda } \\
\text { Hoaglan et. al. }\end{array}$ & $\begin{array}{l}\text { Descreve a percepção da } \\
\text { PrEP e disposição sobre } \\
\text { o uso da medicaçãa entre } \\
\text { homens ue fazem sexo com } \\
\text { homens }\end{array}$ & $\begin{array}{c}\text { Estudp transversal de } \\
\text { coorte/ CAPS: A1 Nivel de } \\
\text { evidência: IV }\end{array}$ & $\begin{array}{c}\text { Foi relatada a consciência } \\
\text { da PrEP em alguns grupos } \\
\text { vulnerávais, percebe-se a es- } \\
\text { cassez da educação em saúde } \\
\text { voltada a promoção acerca } \\
\text { dessa terapia }\end{array}$ \\
\hline
\end{tabular}




\section{artigo}

Ataíde, E.A.O.; Andrade, C.A.A.; Freire, D.A.; Abrão, F.M.S.

Homens que fazem sexo com homens: conhecimento, empoderamento a profilaxia pré-exposição ao vírus da imunodeficiência humana

sendo assim identificados como principais grupos para implementação da PrEP. Foi identificado também o conhecimento limitado à profilaxia pré-exposição e a baixa percepção de risco ao HIV, representando assim desafios a serem abordados ${ }^{17}$.

Assim, vale salientar os avanços em relação ao tratamento ao HIV e, consequente, uma redução significativa ao número de casos de AIDS, além do acesso ao SUS, mas, ainda, há muito o que ser discutido e divulgado, pois números mostram relações sexuais desprotegidas e com mais de um parceiro, e informaçôes deficientes sobre a profilaxia pré-exposição ao HIV para, dessa forma, as pessoas, em especial, Homens que fazem sexo com Homens, se sintam empoderados para realizarem sua prática sexual segura e com isso viver com uma melhor qualidade de vida.

\section{CONCLUSÃO}

O objetivo desse estudo foi alcançado, porém percebe-se a necessidade de ações educativas sobre a profilaxia pré-exposição ao vírus da imunodeficiência adquirida. Sendo imprescindível a liberdade e disponibilidade da diversidade de métodos preventivos, em âmbito individual, e proteção nos diferentes momentos da cadeia de transmissão do HIV. Entretanto, novos meios de terapia que sejam profiláticos não subsistem se não há desdobramentos da equipe multiprofissional na articulação de açốes educativas em populações vulneráveis ao HIV e ao mesmo tempo em que essas medidas são tomadas reforça-se cada vez mais o empoderamento desses indivíduos.

Logo, na perspectiva programática, como já amplamente reconhecido por di- versos atores, o posicionamento dos profissionais de saúde deve ser voltado para promoção e prevenção ao HIV e, sobretudo reforçadores para a melhoria do acesso a PrEP, inclusive aqueles métodos preventivos mais clássicos, como preservativos.

É imprescindível que as evidências encontradas nessa pesquisa possam subsidiar a implementação das políticas públicas nesse campo. Diante dessa realidade, esse conjunto de ações articuladas e harmonizadas nos diversos níveis de atenção, poderá fazer frente às barreiras estruturais que além de reduzir o estigma persistente relativo à orientação sexual irá contribuir também no fortalecimento e direito dos indivíduos de ter acesso a métodos e estratégias de prevenção mais adequada ao seu contexto de vida. -

\section{REFERÊNCIAS}

1. Pereira AJ, Nichiata LYI. A sociedade civil contra Aids: demandas coletivas e politicas públicas. Rev. Ciência e saúde Coletiva, 2011; 16(7):3249-3257

2. Bandeira AG. O envelhecimento e a prevenção do HIV/AIDS: um desafio para os profissionais de saúde. Rev Ciencia e Saúde. 2014; 7(3):115.

3. COLLABORATORS GBD. Estimates of global, regional, and national incidence, prevalence, and mortality of HIV, 1980 - 2015: the Global Burden of Disease Study 2015. The Lancet HIV. 2016;(3):361-387.

4. Brasil. Ministério da Saúde (MS). Coordenação Nacional de DST e Aids. Novos desafios da prevenção da epidemia de HIV/AIDS junto aos homens que fazem sexo com homens (XII Seminário do Programa de Cooperação Técnica Brasil-França). Brasília: MS; 2002

5. Rios LF, Albuquerque AP, Santana WJ, Pereira AF, Júnior CJO. Posições sexuais, estilos corporais e risco para o HIV entre homens que fazem sexo com homens no Recife, Brasil. 2019. Rev Ciência e Saúde Coletiva, 24(3):973-982, 2019

6. Brasil. Ministério da Saúde. Programa Nacional de DST, AIDS e Hepatites Virais. Relatório de progresso da resposta brasileira ao HIV/AIDS (2010-2011) - UNGASS. Brasília: MS; 2012.

7. Kerr L, Mota R, Kendall C, Pinho A, Mello M, Guimarães M, et al. HIV among MSM in a large middle-income country. AIDS. 2013; 27(3):427-435

8. Rocha G, Kerr L, Brito A, Dourado I, Guimarães, M. Unprotected Receptive Anal Intercourse Among Men Who have Sex with Men in Brazil. AIDS Behav 2013; 17(4):1288-1295

9. Lyons A, Pitts M, Grierson J. Versatility and HIV vulnerability: patterns of insertive and receptive anal sex in a national sample of older Australian gay men. AIDS Behav 2013; 17(4):1370-1377

10. Calazans GJ, Pinheiro TF, Mesquita JRC. Vulnerabilidade programática e cuidado público: Panorama das políticas de prevenção do HIV e da Aids voltadas para gays e outros HSH no Brasil. Sex., Salud Soc. 2018,(29):263-293.

11. https://unaids.org.br/prevencao-combinada/acesso em 05/08/19

12. Queiroz AAFLN, Sousa AFL. Fórum PrEP: um debate on-line sobre uso da profilaxia pré-exposição no Brasil. Cad. Saúde Pública 2017; 33(11):2-3

13. URSI, ES. Prevenção de lesões de pele no perioperatório: revisão integrativa da literatura [dissertação]. Ribeirão Preto (SP): Universidade de São Paulo; 2005.

14. Melnyk MB, Fineout-Overholt E, Stillwell SB, Williamson, KM. Evidence-Based Practice: Step by step. American Journal of Nursing. 2010; 110(5): 41-7.

15. Werner RN, et al.,2018. Knowledge and use of HIV pre-exposure prophylaxis among men who have sex with men in Berlin - A multicentre, cross-sectional survey. https://journals.plos. org/plosone/article/figure?id=10.1371/journal.pone.0204067. t006Acesso em 19 de junho de 2019.

16. Alia A Al-Tayyib 1, Mark W Thrun, Jason S Haukoos, Paredes N Eugene, 2014. Knowledge of pre-exposure prophylaxis (PrEP) for HIV prevention among men who have sex with men in Denver, Colorado. Disponivel em: https://www.ncbi.nlm.nih.gov/pmc/articles/PMC4066993/. Acesso em 19 de junho de 2019.

17. Hoaglan. B, et al., 2017. Pre-exposure prophylaxis for men and transgender women who have sex with men in Brasil: Opportunities and challenges. Disponivel https://pubmed.ncbi.nlm.nih. gov/28418232/. Acesso em 19 de junho de 2019. 